\title{
Feeding of the elliptic flow by hard partons
}

\author{
Boris Tomášik ${ }^{1,2}$ and Péter Lévai ${ }^{3}$ \\ ${ }^{1}$ Univerzita Mateja Bela, 97401 Banská Bystrica, Slovakia \\ ${ }^{2}$ Czech Technical University in Prague, FNSPE, 11519 Prague, Czech Republic \\ ${ }^{2}$ MTA KFKI RMKI, Budapest, Hungary \\ E-mail: boris.tomasik@umb.sk
}

\begin{abstract}
We propose that in nuclear collisions at the LHC the elliptic flow may get a contribution from leading hard and semihard partons which deposit energy and momentum into the hydrodynamic bulk medium. The crucial effect is that these partons induce wakes which interact and merge if they come together. The contribution to the integrated elliptic flow is estimated with the help of a toy model to about $25 \%$ of the observed value and shows strong event-by-event fluctuations.
\end{abstract}

PACS numbers: 25.75.-q, 25.75.Ld

\section{Introduction}

The azimuthal anisotropy of hadron production in ultrarelativistic nuclear collisions, known under the term elliptic flow, has proved to be an important observable [1, 2, 3. In particular, its observed size lead to conclusions that concern the time of thermalisation [4, 5] and the influence of shear and bulk viscosity [6, 7, 8, 9, 10, 11]. To get to these important and interesting characteristics of the bulk matter one must, however, reasonably quantify the influence of other effects [12, 13, 14]. Among them there is our ignorance about the actual initial conditions for the hydrodynamic evolution of the fireball as well as the role of fluctuations in the initial conditions [15, 16, 17]. Also, the transition from dense hydrodynamic matter to more dilute hadronic gas, which could slip out of equilibrium and later freezes-out, may also impact the momentum anisotropy $[18,19,20,21,22$. In this paper we describe a contribution to the elliptic flow which may be important at the LHC energy and possibly also at RHIC.

The standard interpretation of the elliptic flow is that it is caused by the anisotropy of the pressure gradients within the excited matter. They are larger in the direction of the impact parameter, which is usually called 'in-plane' direction. (The other transverse direction is denoted 'out-of-plane'.) The anisotropic pressure gradients lead to different accelerations of the collective flow in the different directions. The fireball then finally expands faster in the in-plane direction. Therefore, more hadrons are emitted in this direction and their transverse momentum spectra are flatter than in the out-of-plane direction. The measured size of this anisotropy, its dependence on the transverse 
momentum and on particle species depend on many effects. The one among them we want to focus at is the dependence on viscosity, since one would like to extract this transport coefficient from the data [14].

Thus, if there is an additional cause for the azimuthal anisotropy of hadron production, this might influence the statements concerning fast thermalisation and maybe even modify the conclusion about low viscosity. In this paper, we consider such a mechanism.

At the LHC, jets and minijets are produced copiously. Their energy loss when crossing the deconfined matter is so huge that only a few of them appear as distinguished jets [23, 24, 25, 26]. They rather transfer their energy and momentum into the bulk matter and initiate collective phenomena there [27, 28, 29, 30, 31, 32, 33, 34, 35, 36]. Momentum must be conserved and thus the momentum of the hard parton must be transformed into momentum of a stream of matter (a wake), a Mach cone wave, or something like this. Much interest is currently devoted to such effects.

A question arises, what would be the result of many such streams if they all are initiated in the fireball? Some of these streams could merge and either cancel or flow in a new direction so that energy and momentum are conserved. Original hard partons are produced with no preferred transverse direction. The first expectation would be that the large number of fluid streams they initiate cancel out in some way and in the end there remains just thermalised matter with some energy density and no macroscopic flow. However, in non-central collisions the argument might not be so straightforward. The streams have random directions, but their spatial distribution is not isotropic, since it is given by the initial collision geometry. In the in-plane direction the fireball is narrower. Thus there is a good chance that two streams having finite width and flowing in the out-of-plane direction will meet (see Figure 1). On the other hand, streams could more easily pass each other without interacting when produced in the in-plane direction. Thus - very grossly - streams perpendicular to the reaction plane cancel each other while those flowing in directions parallel to the reaction plane survive. As a result, the flow of bulk matter receives some feeding from hard partons and this feeding exhibits signs of a positive elliptic flow.

Note that the scenario proposed here is related in its spirit to hydrodynamic simulations with hot spots in the initial conditions which are simulated on the eventby-event basis [15, 16, 37, 38, 39, 40]. Contribution to elliptic flow of the bulk from semi-hard partons has also been considered in [35, 36].

Obviously, this argument is very schematic and to make it more sound one would need to integrate over all possible directions of the streams. The proper way to do so would be the use of a hydrodynamic simulation with included energy and momentum deposition from hard partons. At present, such simulations are not available due to technical complexity. Therefore, in order to obtain simple estimates of the possible size of the effect, we rather construct a simple toy model, which resembles the effect.

In our toy model which is introduced in the next Section, we represent the flowing streams by flying blobs of matter with specified size. If two blobs meet, they merge into a 

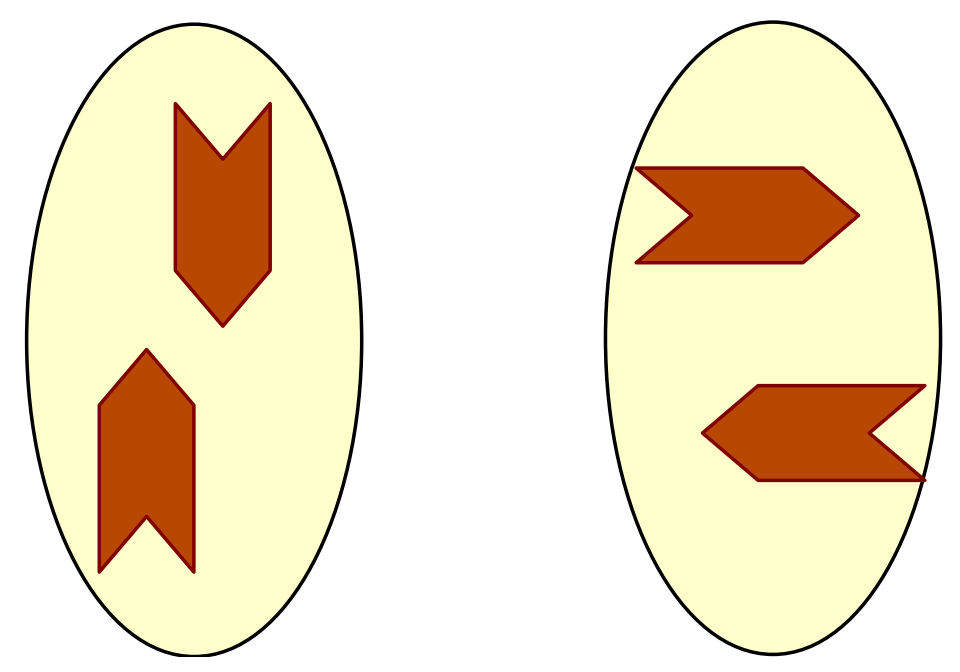

Figure 1. Illustration of the likeness or unlikeness of two streams to meet. Left: two streams flowing in the out-of-plane direction are likely to meet. Right: as the fireball is elongated out of the reaction plane, two streams which flow in the in-plane direction have more space to pass each other without merging.

heavier blob which moves so that energy and momentum are conserved. Originally, many blobs are generated. Their number and momenta correspond to the expected number and momenta of hard partons. In the end, after merging of all blobs which should have merged, they all evaporate pions. In Section 3 we then analyse the distributions of these pions. It turns out that about $25 \%$ of the observed integrated elliptic flow may be due to our effect. We also find that this contribution is very strongly fluctuating. Conclusions are summarised in Section 4 .

\section{The toy model}

Streams within the fluid are represented by blobs. For each blob first its four-velocity is generated. The momentum is generated according to the calculated distributions of the produced hard partons in transverse momentum and rapidity. For rapidity distributions at LHC and RHIC we assume that they are uniform in the central two units of rapidity.

\subsection{Momentum distribution}

Transverse momentum spectra have been calculated and the differential cross section for gluon production in proton-proton collision was parametrised as

$$
E \frac{d \sigma_{N N}}{d p^{3}}=\frac{1}{2 \pi} \frac{1}{p_{t}} \frac{d \sigma_{N N}}{d p_{t} d y}=\frac{B}{\left(1+p_{t} / p_{0}\right)^{n}}
$$

where $p_{0}, B$, and $n$ are parameters. The parametrisation works fine in the $p_{t}$ interval from 2.5 to $12 \mathrm{GeV} / c$. Calculated spectra deviate from this parametrisation for higher $p_{t}$. Note, however, that production of jets at such high $p_{t}$ is rare and thus does not contribute much to the total yield and can be assumed to have small effect on the bulk 
when large number of collisions is analysed. For a simulation at LHC energies we chose $B=14.7 \mathrm{mbarn} / \mathrm{GeV}^{2}, p_{0}=6 \mathrm{GeV}$, and $n=9.5$.

Now we calculate the total number of blobs in a non-central symmetric collision of two nuclei with mass numbers $A$ at the impact parameter $b(b=|\vec{b}|)$. The cross-section for the production of the leading particle with $p_{t}$ larger that $p_{m}$ is then obtained by integrating eq. (1)

$$
\sigma\left(p_{m}\right)=\int_{p_{m}}^{\infty} \int_{y_{\min }}^{y_{\max }} \frac{d \sigma_{N N}}{d p_{t} d y} d p_{t} d y .
$$

The mean total number of leading particles with $p_{t}>p_{m}$ is then

$$
N_{j}\left(p_{m}, b\right)=\frac{A^{2} T_{A A}(b) \sigma\left(p_{m}\right)}{1-\left(1-T_{A A}(b) \sigma\left(p_{m}\right)\right)^{A^{2}}} .
$$

In the last equation we introduced the overlap function

$$
\begin{aligned}
& T_{A A}(b)=\int_{\text {overlap }} T_{A}(\vec{r}) T_{A}(\vec{r}-\vec{b}) d^{2} \vec{r}, \\
& T_{A}(\vec{r})=2 \rho_{0} \sqrt{R_{A}^{2}-r^{2}} .
\end{aligned}
$$

where $T_{A}(\vec{x})$ is the nuclear thickness function. The radius of the nucleus is $R_{A}$ and for the sake of our estimates we have assumed very simple profile with constant nuclear density $\rho_{0}$.

\subsection{Evolution of blobs}

The blobs represent streams of bulk matter and carry the momentum of the leading partons. Once the momentum of the blob is given, for the simulation we need to determine the velocity of the blob. It will be close to $c$ since all energy of the partons is basically due to momentum. Technically, we choose a very small off-shell mass of $m=1 \mathrm{GeV}$. Then, the velocity of a blob with transverse momentum $p_{t}$ and rapidity $y$ is

$$
v^{\mu}=\left(m_{t} \cosh y, p_{t} \cos \phi, p_{t} \sin \phi, m_{t} \sinh y\right),
$$

where $m_{t}=\sqrt{p_{t}^{2}+m^{2}}$ and the azimuthal angle $\phi$ is generated randomly from a uniform distribution.

Jets are produced in pairs which are roughly back to back in the transverse plane (but not longitudinally) so that the total transverse momentum vanishes. However, in the parametrisations of the leading parton differential cross section we have assumed some broadening of the transverse momentum due to intrinsic $\left\langle k_{t}^{2}\right\rangle$. Since this is an initial state effect, it follows that the total transverse momentum of the hard parton pair should be of the order $\left\langle k_{t}^{2}\right\rangle$. Following this geometry we find that the away-side parton is not directed precisely in the oposite way but may deviate from this direction by some angle $\alpha$ of the order

$$
\alpha^{2} \simeq \frac{\left\langle k_{t}^{2}\right\rangle}{p_{t}^{2}}
$$


Here, we have assumed that $\left\langle k_{t}^{2}\right\rangle \ll p_{t}^{2}$. Thus the azimuthal angle of the generated away side leading parton shall deviate from the opposite direction by this $\alpha$, which will be generated from a Gaussian distribution with the width $\sqrt{\left\langle k_{t}^{2}\right\rangle / p_{t}^{2}}$. In case of small $p_{t}$ this may become a large number. Thus if $\left\langle k_{t}^{2}\right\rangle / p_{t}^{2}>0.7$ we shall fix the width to 0.7 .

The transverse positions at which the blobs start moving are distributed according to the density of binary nucleon-nucleon collisions

$$
\rho_{b}(\vec{r})=T_{A}(\vec{r}) T_{B}(\vec{r}-\vec{b})
$$

The time at which the blob is created is delayed by some formation time $\tau_{0}$ (set usually to $0.6 \mathrm{fm} / c$ ) multiplied by $\gamma$. The longitudinal position is then $\tau_{0} v_{3}$. Longitudinal rapidity is chosen from a uniform distribution.

Two blobs merge when they approach each other in their pair CMS closer than $2 R_{b}$, where $R_{b}$ is a model parameter which we will vary in our simulations. In this formulation it is the size of a blob but it actually represents the radius of the fluid stream. In the merger a new blob with the same size and a higher energy content is created. It will move with velocity $v$ and the direction of the velocity is chosen such that energy and momentum are conserved.

When there are no more mergers the blobs evaporate into pions. In the rest frame of the blob, pions emitted by that blob are distributed thermally with a kinetic temperature $T$. In our simulation we choose $\mathrm{T}=160 \mathrm{MeV}$.

\section{Elliptic flow}

The elliptic flow coefficient $v_{2}$ is defined as the second order Fourier coefficient in the decomposition of the azimuthal single-particle distribution [2]

$$
P(\phi)=\frac{d N}{d \phi}=N_{0}\left(1+2 v_{2} \cos \left[2\left(\phi-\phi_{0}\right)\right]+\ldots\right),
$$

where $\phi_{0}$ is the angle of the event plane. We have written this relation for midrapidity so that certain symmetry constraints apply. Since in our simulation we know the orientation of the reaction plane, we can always use the coordinate frame in which $\phi_{0}=0$ and determine $v_{2}$ from

$$
v_{2}=\frac{\int_{0}^{2 \pi} P(\phi) \cos (2 \phi) d \phi}{\int_{0}^{2 \pi} P(\phi) d \phi} .
$$

We measure $v_{2}$ in each event as the average of $\cos (2 \phi)$ over all particles and then we take the average over all events.

In Figure 2 we show the azimuthal distributions summed over all simulated events. For the most central classes we simulated more than 150,000 events and for centralities over $50 \%$ the samples count 100,000 events. For the analysis we accepted pions in rapidity window from -1 to 1 .

In the histograms we clearly see that the production of final state hadrons is correlated with the reaction plane. Thus we deal with true flow signal and not just 

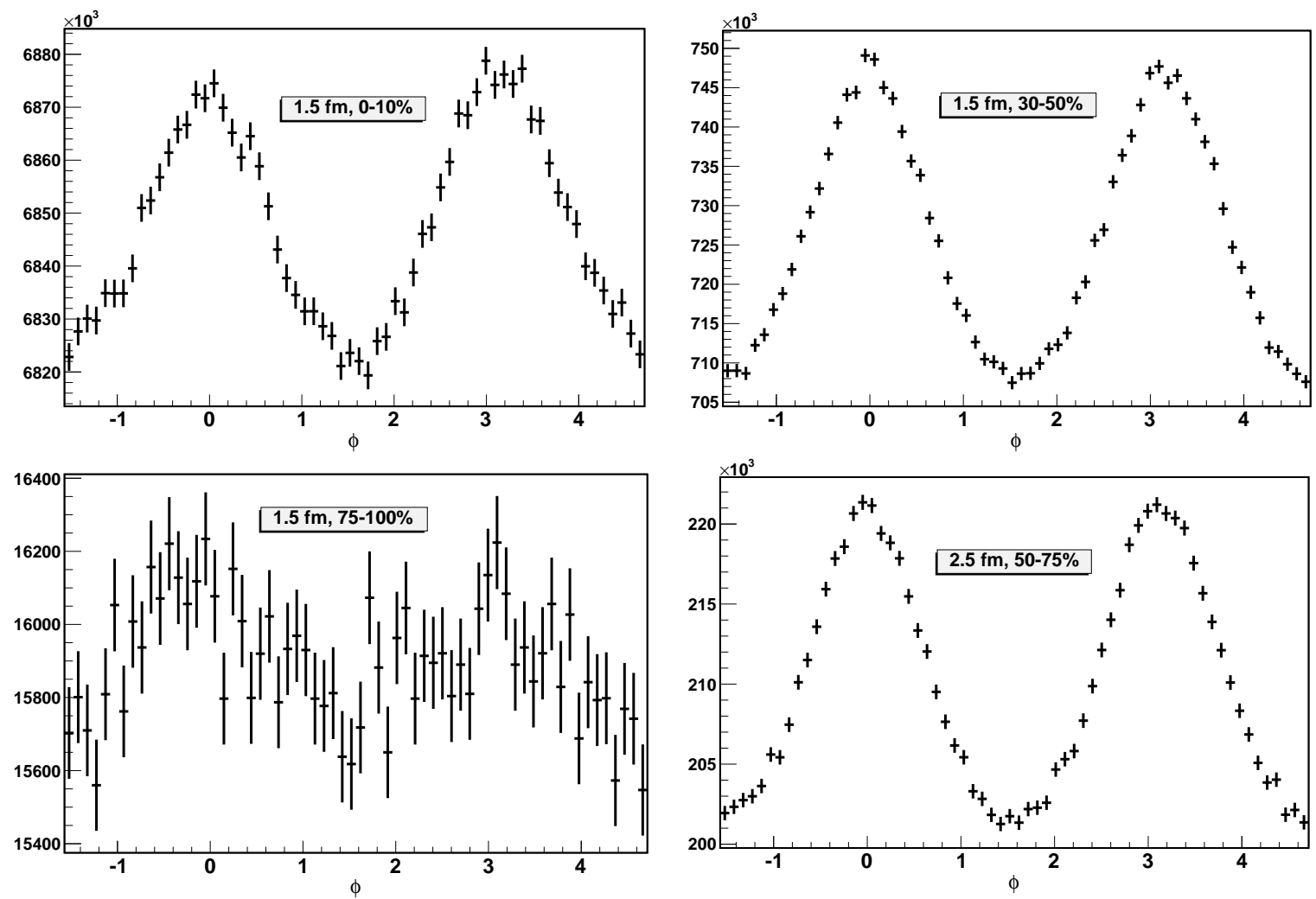

Figure 2. Histograms of the azimuthal distributions of pions integrated over $p_{t}$. Different panels correspond to different radii of the streams $R_{b}$ and different centrality classes, as indicated in the panels.

with a non-flow effect stemming from correlation of individual hadrons with each other. The difference between results of simulations with two chosen radii of streams is not very large.

In order to better see how the anisotropy depends on centrality and the size of the streams we plot in Figure 3 the elliptic flow parameter $v_{2}$ as function of centrality. As expected, $v_{2}$ increases when departing from central events towards more peripheral. This growth turns into decrease for the most peripheral collisions due to lower density of streams where the chance of mergers is smaller. The integrated $v_{2}$ in our simulations reaches up to about 0.02 . Note that this amounts up to $25 \%$ of the integrated $v_{2}$ measured in $\mathrm{Pb}+\mathrm{Pb}$ collisions at 2.76 ATeV [41] and a similar share of the elliptic flow at RHIC [42, 43]. Thus we want to conclude that the proposed effect of momentum feeding from hard leading partons with subsequent stream interaction may contribute considerably to the observed elliptic flow.

We also found in the simulation that $v_{2}$ exhibits large fluctuations of the order of mean value or even more in some cases. This follows from the nature of the effect which crucially depends on the mergers of streams and vanishes if no such effect occur in a given event. The fluctuation might possibly be milder if complete hydrodynamic evolution is added to the picture. Nevertheless, we expect that one of the signatures of 


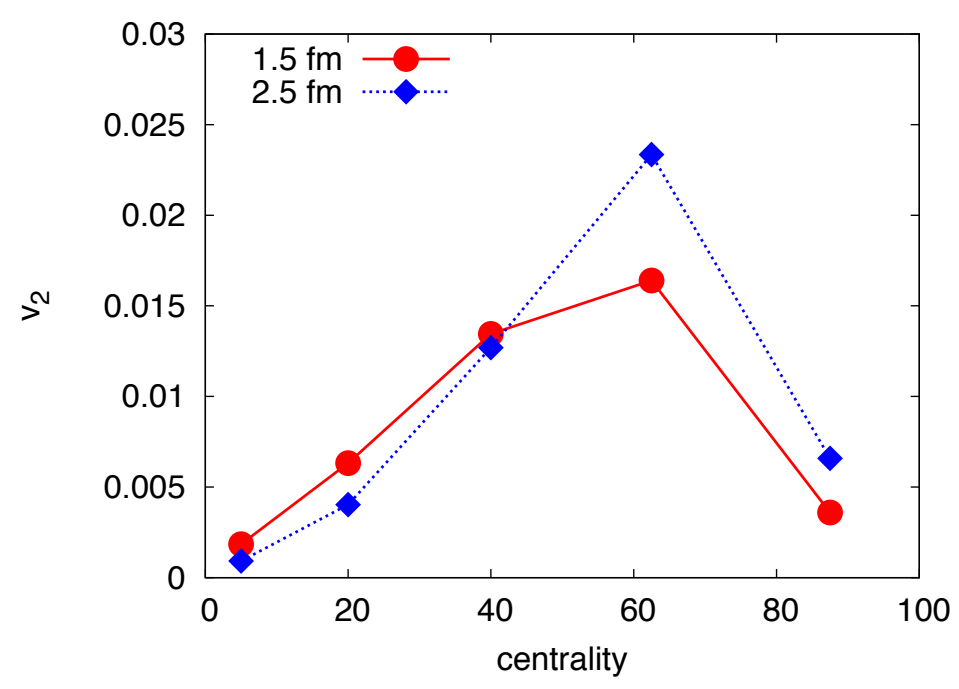

Figure 3. Centrality dependence of $v_{2}$ for different radii of the streams: $R_{b}=1.5 \mathrm{fm}$ (solid red line), $R_{b}=2.5 \mathrm{fm}$ (dotted blue line).

the effect discussed here will be enhanced $v_{2}$ fluctuations.

\section{Conclusions}

Our study qualitatively shows that in nuclear collisions at the LHC relevant effect for momentum anisotropy may be the transfer of momentum from the leading hard partons to hydrodynamic medium. Crucial ingredient is the interaction of streams induced in the bulk medium. They may merge and flow together. The net effect is the deflection of some of the streams from the out-of-plane direction into the in-plane direction.

The toy model simulation amounted to $25 \%$ of the elliptic flow that is measured in $\mathrm{Pb}+\mathrm{Pb}$ collisions at the LHC. The conclusion is that the effect may be important and should be included in simulations aimed at extraction of bulk matter properties by comparing to experimental data. So far, simulations with one jet depositing momentum into bulk matter have been performed [33]. However, to our best knowledge no hydrodynamic simulations exist, where the effect of energy and momentum feeding from many hard partons would be investigated.

Acknowledgements In its initial phase, this work was supported by the Hungaro-Slovak collaboration grant No. SK-HU-029-06 (SK) and SK 20/2006 (HU). BT ackowledges support via grants Nos MSM 6840770039, and LC 07048 (Czech Republic). This work was partially supported by the Agency of the Slovak Ministry of Education for the Structural Funds of the EU, under project ITMS:26220120007. PL acknowledges the 
support of OTKA grant No 77816.

\section{References}

[1] J. Y. Ollitrault, Phys. Rev. D 46 (1992) 229.

[2] S. Voloshin and Y. Zhang, Z. Phys. C 70 (1996) 665 arXiv:hep-ph/9407282.

[3] P. F. Kolb, J. Sollfrank and U. W. Heinz, Phys. Rev. C 62 (2000) 054909 arXiv:hep-ph/0006129].

[4] U. W. Heinz and P. F. Kolb, Nucl. Phys. A 702 (2002) 269 arXiv:hep-ph/0111075.

[5] P. Bożek and I. Wyskiel-Piekarska, Phys. Rev. C 83 (2011) 024910 arXiv:1009.0701 [nucl-th]].

[6] T. Hirano, U. W. Heinz, D. Kharzeev, R. Lacey and Y. Nara, Phys. Lett. B 636 (2006) 299 arXiv:nucl-th/0511046.

[7] H. J. Drescher, A. Dumitru, C. Gombeaud and J. Y. Ollitrault, Phys. Rev. C 76 (2007) 024905 arXiv:0704.3553 [nucl-th]].

[8] P. Romatschke and U. Romatschke, Phys. Rev. Lett. 99 (2007) 172301 arXiv:0706.1522 [nucl-th]].

[9] D. A. Teaney, arXiv:0905.2433 [nucl-th].

[10] H. Masui, J. Y. Ollitrault, R. Snellings and A. Tang, Nucl. Phys. A 830 (2009) 463C arXiv:0908.0403 [nucl-ex]].

[11] H. Song and U. W. Heinz, Phys. Rev. C 81 (2010) 024905 arXiv:0909.1549 [nucl-th]].

[12] M. Luzum and P. Romatschke, Phys. Rev. C 78 (2008) 034915 [Erratum-ibid. C 79 (2009) 039903] arXiv:0804.4015 [nucl-th]].

[13] P. Bożek, Phys. Rev. C 81 (2010) 034909 arXiv:0911.2397 [nucl-th]].

[14] C. Shen, U. Heinz, P. Huovinen and H. Song, Phys. Rev. C 82 (2010) 054904 arXiv:1010.1856 [nucl-th]].

[15] R. Andrade, F. Grassi, Y. Hama, T. Kodama and O. J. Socolowski, Phys. Rev. Lett. 97 (2006) 202302 arXiv:nucl-th/0608067.

[16] B. Schenke, S. Jeon and C. Gale, Phys. Rev. Lett. 106 (2011) 042301 arXiv:1009.3244 [hep-ph]].

[17] H. Petersen and M. Bleicher, Phys. Rev. C 81 (2010) 044906 arXiv:1002.1003 [nucl-th]].

[18] H. Petersen, J. Steinheimer, G. Burau and M. Bleicher, Eur. Phys. J. C 62 (2009) 31.

[19] T. Hirano, P. Huovinen and Y. Nara, Phys. Rev. C 83 (2011) 021902 arXiv:1010.6222 [nucl-th]].

[20] T. Hirano, P. Huovinen and Y. Nara, arXiv:1012.3955 [nucl-th].

[21] H. Song, S. A. Bass, U. W. Heinz, T. Hirano and C. Shen, arXiv:1101.4638 [nucl-th].

[22] H. Song, S. A. Bass and U. W. Heinz, arXiv:1103.2380 [nucl-th].

[23] J. Adams et al. [STAR Collaboration], Phys. Rev. Lett. 91 (2003) 072304 arXiv:nucl-ex/0306024].

[24] G. Aad et al. [Atlas Collaboration], Phys. Rev. Lett. 105 (2010) 252303 arXiv:1011.6182 [hep-ex]].

[25] S. Chatrchyan et al. [CMS Collaboration], arXiv:1102.1957 [nucl-ex].

[26] K. Aamodt et al. [ALICE Collaboration], Phys. Lett. B 696 (2011) 30 arXiv:1012.1004 [nucl-ex]].

[27] L. M. Satarov, H. Stoecker and I. N. Mishustin, Phys. Lett. B 627 (2005) 64 arXiv:hep$\mathrm{ph} / 0505245$.

[28] J. Casalderrey-Solana, E. V. Shuryak and D. Teaney, J. Phys. Conf. Ser. 27 (2005) 22 [Nucl. Phys. A 774 (2006) 577] arXiv:hep-ph/0411315].

[29] J. Ruppert and B. Müller, Phys. Lett. B 618 (2005) 123 arXiv:hep-ph/0503158.

[30] T. Renk and J. Ruppert, Phys. Rev. C 73 (2006) 011901 arXiv:hep-ph/0509036.

[31] R. B. Neufeld, Phys. Rev. D 78 (2008) 085015 arXiv:0805.0385 [hep-ph]].

[32] R. B. Neufeld and T. Renk, Phys. Rev. C 82 (2010) 044903 arXiv:1001.5068 [nucl-th]].

[33] B. Betz, J. Noronha, G. Torrieri, M. Gyulassy, I. Mishustin and D. H. Rischke, Phys. Rev. C 79 (2009) 034902 arXiv:0812.4401 [nucl-th]].

[34] E. Shuryak, arXiv:1101.4839 [hep-ph].

[35] R.C. Hwa, Phys. Lett. B 666 (2008) 228.

[36] C. B. Chiu, R. C. Hwa, C. B. Yang, Phys. Rev. C 78 (2008) 044903.

[37] H. Holopainen, H. Niemi and K. J. Eskola, Phys. Rev. C 83 (2011) 034901 arXiv:1007.0368 [hep-ph]]. 
[38] H. Petersen, C. Coleman-Smith, S. A. Bass and R. Wolpert, J. Phys. G 38 (2011) 045102 arXiv:1012.4629 [nucl-th]].

[39] M. S. Borysova, I. A. Karpenko and Yu. M. Sinyukov, arXiv:1102.2084 [nucl-th].

[40] F. G. Gardim, F. Grassi, Y. Hama, M. Luzum and J. Y. Ollitrault, arXiv:1103.4605 [nucl-th].

[41] K. Aamodt et al. [The ALICE Collaboration], Phys. Rev. Lett. 105 (2010) 252302. arXiv:1011.3914 [nucl-ex]].

[42] B. I. Abelev et al. [STAR Collaboration], Phys. Rev. C 77 (2008) 054901 arXiv:0801.3466 [nucl$\mathrm{ex}]$.

[43] S. Afanasiev et al. [PHENIX Collaboration], Phys. Rev. C 80 (2009) 024909 arXiv:0905.1070 [nucl-ex]]. 